\title{
Sensorial Surfaces - Integrated Sensors for Component- Based Condition Monitoring
}

\author{
Dr. Cord Winkelmann ${ }^{1}$ \\ ${ }^{1}$ Winkelmann Mikrosysteme, Otto-Hahn-Allee NW1, 28359 Bremen, Germany, \\ Info@Winkelmann-Mikrosysteme.de
}

\begin{abstract}
:
The advent of cyber-physical systems and the internet of things will change the way automation and condition monitoring works. Today's vertically integrated production and monitoring systems will be linked horizontally - not only within single corporations but also across companies in different fields. The paper describes how automation systems and especially condition monitoring systems might look in the future and demonstrates this with the help of five use cases. Additionally, simulation and measurement results of the development of a condition monitoring system for a linear guide are presented. The deformations were carried out in Comsol Multiphysics and show that the measurement of the magnitude as well as the direction of a force acting on the linear guide can be measured. These results were confirmed in practical tests.
\end{abstract}

Key words: Condition Monitoring, Sensorial Surfaces, Sensor Integration, Internet of Things, CyberPhysical Systems, Industrie 4.0

\section{Introduction}

Condition monitoring plays a major role in monitoring the status of large machines. However, it is not always an easy task to deduce the cause of a failure from the measured data.

This paper looks into a paradigm-change of automation, caused by cyber-physical systems. The hierarchical concept of the automation pyramid with its vertical integration will shift to a horizontal integration in the future.

One important aspect of component-based condition monitoring is the understanding of a sensor. Today, a typical sensor like an accelerometer consists of different mechanical and electrical components. The actual sensor element, responsible for converting a physical quantity (e.g. acceleration) into an electrical signal, is just one of the sensor's components.

In cyber-physical systems however, the sensor element becomes an integral part of the mechanical component to be monitored. Thus, the mechanical component becomes the sensor itself. This concept is described in further detail in the sections about condition monitoring today and tomorrow.

\section{Automation Today}

Figure 1 shows the automation system pyramid that represents the automation implementation of today's companies. It is a hierarchical system with the sensor/actuator-level on its base and the enterprise level on the top.

On the enterprise level, enterprise-resourceplanning (ERP) takes place. This task includes the planning of resources like capital, staff, materials and IT-systems for example. But also a coarse production planning and purchase order processing are located in this level.

Manufacturing execution systems (MES) are used on the management level of the automation system pyramid. In contrast to the ERP systems, it is directly coupled to the

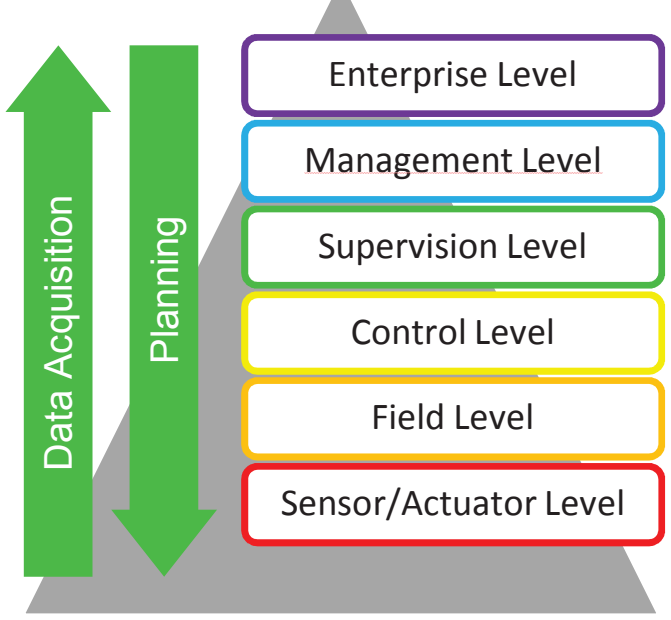

Fig. 1. Automation system pyramid. 
supervision level and controls the production in "soft" real time. The fine planning of production, material and quality management take place on this level.

The supervision level is a typical SCADA(supervisory control and data acquisition) or PCS- (process control system) level. Characteristically, complete facilities or sites like a refinery are supervised on this level. An important task on this level is the archiving of measurement data.

Programmable logic controllers (PLCs) are used on the control level to control the actual production machine. From this level on, "hard" real-time is needed to avoid unintended operations.

The field or device level incorporates sensors, actuators and drive systems as whole systems. A typical example could be an industrial robot or the drive system of a conveyor belt.

The sensor/actuator level is located below. In contrast to the field level, sensors and actuators here mean the actual sensor element like a switch, pushbutton, feeler, for example. These elements are mostly incorporated into devices of the field level as motor encoders or end switches and are often hard-wired.

\section{Condition Monitoring Today}

Condition monitoring today takes place mostly on the field level. Most common applications are the monitoring of temperatures and vibrations i.e. accelerations.

Figure 2 shows the implementation of a typical condition monitoring system. In case of a MEMS- (micro-electro-mechanical-system) accelerometer, the manufacturing process of the sensor starts with silicon wafer. On this wafer the actual sensor element is manufactured, consisting of mechanical and electrical structures. For better handling and to protect the sensor structures from harsh environmental conditions, the chip is housed subsequently. Finally, the sensor can be mounted on a machine, a gear box for example. It detects changes in the vibration level of the machine. This vibration level changes due to wear and thus the time of failure can be determined. Typically, wear can be detected a few days or weeks before failure.
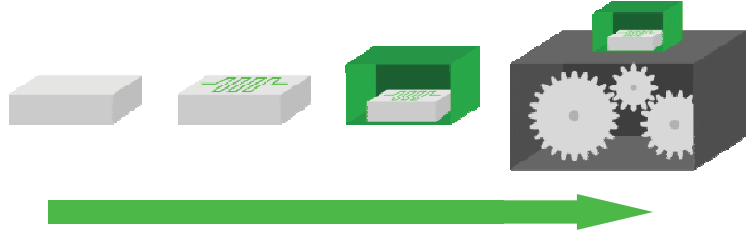

Fig. 2. Typical condition monitoring system.
The sensor is located in the field level and sends its data to the control or supervision level, but not higher.

\section{Automation Tomorrow}

With the beginning advent of the internet of things (IOT) and cyber-physical systems (CPS), more computing power becomes available at levels, where it was unthinkable a couple of years ago. In contrast to embedded systems, cyber-physical systems emphasize highly on a link between the computational and physical elements.

Industrie 4.0 is a future project within the hightech strategy of the cabinet of Germany and incorporates concepts such as CPS, IOT and the internet of services (IOS) [1][2]. In the United States of America, the Smart Manufacturing Leadership Coalition (SMLC), a non-profit organization of manufacturing practitioners, suppliers, technology companies, manufacturing consortia, universities, government agencies and laboratories are working on similar goals [3].

In this future concept, the hierarchical concept of the automation pyramid is not easily applicable. In CPS-based automation the different tasks reside on more or less equal levels as shown in figure 3. The different tasks communicate via internet and are subject to certain rules. Because the different levels are able to communicate directly, all data becomes instantly available to every level and the planning of tasks can be negotiated directly between the levels, unlike the bottom-up or topdown approach of the automation pyramid.

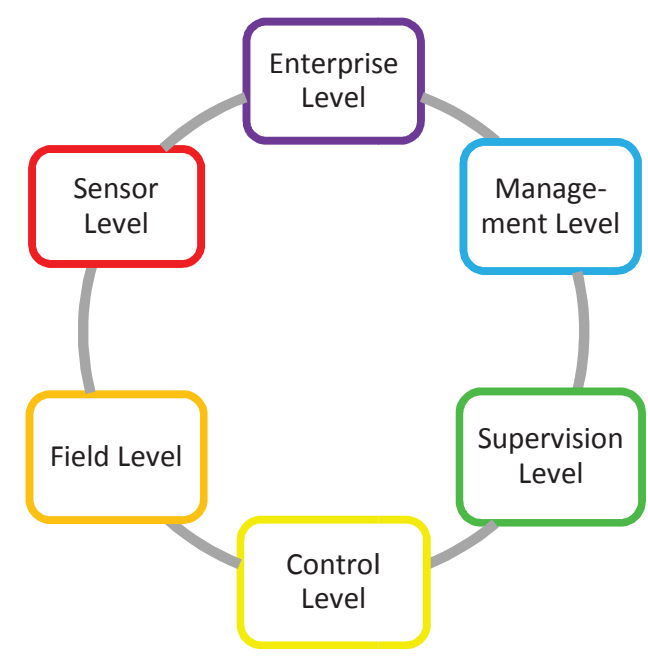

Fig. 3. CPS-based automation 

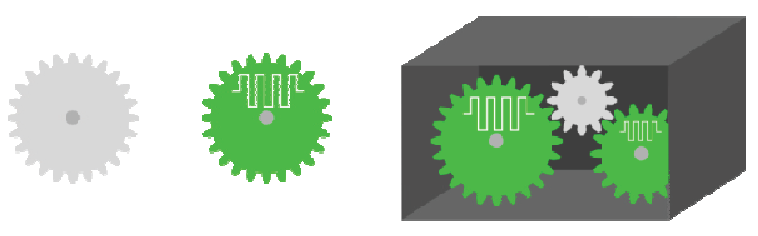

Fig. 4. Direct measurement of loads on the part to be monitored.

\section{Condition Monitoring Tomorrow}

Sensor integration and especially sensorial surfaces offer new opportunities for condition monitoring systems. Sensor integration means that single sensor elements are embedded in the material matrix of the part or component to be monitored [4].

Sensorial surfaces are a pre-stage to sensor integration. Here, the single sensor elements are manufactured directly on the surface of the part to be monitored as depicted in figure 4 . This means, that the single sensor elements and the part form a single unity [5][6] and thus the part itself becomes "self-aware" about its own status. In contrast to classical condition monitoring as described above, the primary sources causing wear and failure (like forces, torque or temperatures) can be monitored constantly with a high spacial and time resolution. That way, the complete history of crucial physical influences is known and better predictions about the remaining useful life of the part can be achieved.

Since the sensorial surface becomes an integral part of a single physical component of the machine, the monitoring system shifts from the field level to the sensor/actuator level. Since it can communicate directly with the other levels according to the concept of CPS-based automation, new use cases and business models will follow.

\section{Use Case 1: Communication within a corporation}

As described before, the mechanical component that is equipped with a componentbased condition monitoring system, can communicate directly with all other levels within a corporation (figure 5). It will, for example, tell the control unit of a machine about the magnitude of forces acting on the part. This data can also be used on the management level for quality management, because a degrading tool leads to a lower processing quality. Thus, former quality assurance processes that were located downstream in the manufacturing process, will be integrated into the manufacturing process itself, leading to a faster reaction time.

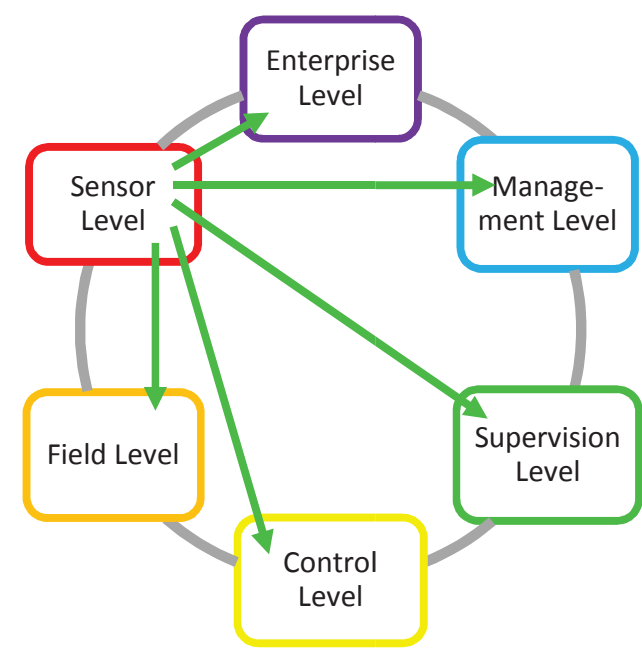

Fig. 5. Communication within a corporation.

\section{Use case 2: Inter-Component communication}

In many cases, companies use more than one machine of the same type in parallel. This gives the sensor systems the opportunity to profit from events on other machines by automated machine learning techniques. In figure 6, components A communicate with each other within Corporation 1 (left) but not necessarily with a component $B$ because components $A$ and $B$ are very different parts (e.g. a rotary bearing and a gear). However, Corporation 1 and Corporation 2 (left and right) can profit from sharing data on the sensor level, if the same or similar components are used in both companies, even in different machines. Thus, both companies, although they might be in completely different business fields, can improve the prediction of downtimes of their own machines.

\section{Use case 3: Communication between a producing corporation and a maintenance- service provider}

If the sensor system predicts a failure of the monitored part in the near future, machine maintenance has to be scheduled. In many companies, the maintenance service is carried

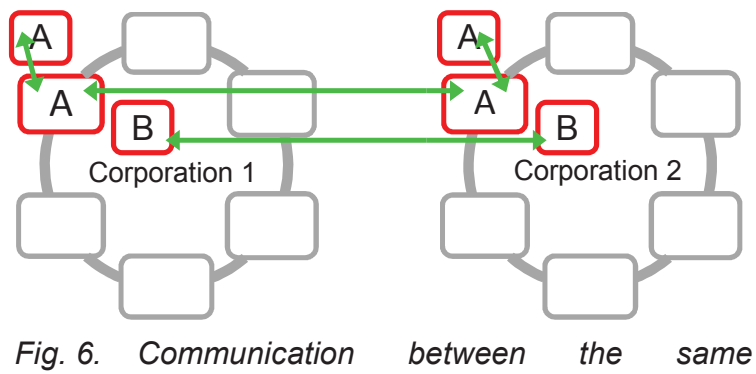
components within and across companies. 


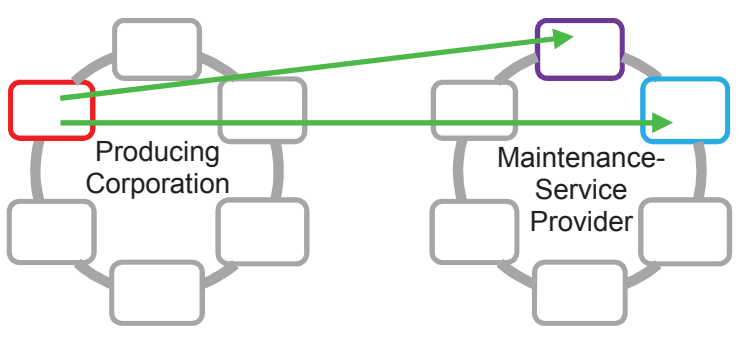

Fig. 7. Communication between a producer (left) and an external maintenance and repair company (right).

out by external contractors (figure 7). The component-based condition monitoring system of the customer (left) will tell the contractor (right) about the upcoming procedure. Since the maintenance-service provider knows what part will need to be serviced, it can plan its staff, order and prepare needed spare parts and schedule a service date with the customer. Through this streamlined process the customer saves machine-downtimes and the contractor can plan its staff better and shrink its spare-part storage.

\section{Use case 4: Communication between a producing corporation and the manufacturer of machining tools}

Figure 8 shows the interaction between a producing company (left) and the manufacturer of a machining tool or manufacturer of machine components.

Single sensorial components send key values to the designer of the machine, e.g. a machining tool. The machining tool manufacturer receives sensor data on the enterprise, management and sensor level. On the enterprise level it can see how a certain machine part is performing. This data can be used as marketing data about machine durability and downtimes, for example. Another use will be the warranty management. The manufacturer can guarantee the functioning of the machine under certain conditions and protect itself from unjustified warranty claims.

On the management level, the data can be used to improve the construction of new

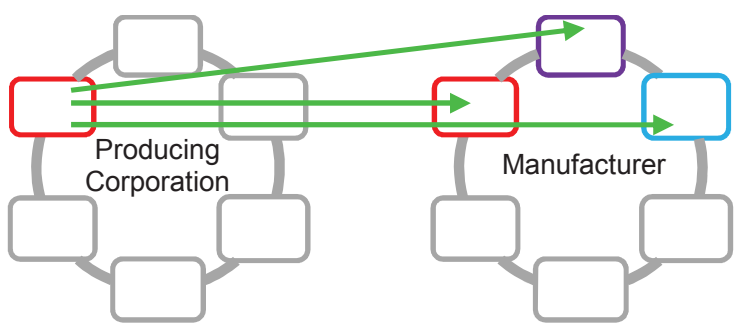

Fig. 8. Communication between a producer (left) and the manufacturer of a machine tool or the manufacturer of machine components (right). machines, for example. It can influence the selection of machine components based on reliability data gathered under real manufacturing conditions.

\section{Use case 5: Communication between a producing corporation and the manufacturer machine components}

Manufacturers of machine components such as bearings, linear guides or gears can profit in the same way as the manufacturers of complete machines. Here, especially the communication on the sensor level can be utilized. Since the manufacturer gets data from very different machines, it can further improve its product and the underlying construction models.

\section{Sensorial Linear Guide}

Winkelmann Mikrosysteme is currently developing a condition monitoring system for linear guides. The goal is to provide a compact sensor system that measures forces on the rail of the linear guide and does the necessary calculations to determine the remaining useful life of the guide.

To analyze the deformation of the rail auf the linear guide, FEM simulations were carried out with Comsol Multiphysics. For this, rails of the TSXxx family from Schaeffler were analyzed, where $x x$ stands for the size of the rail $(35,45$, 55 , and 65). The dxf-models were loaded into Comsol and forces, acting on the running surface were set as pressures (figure 9). The forces included the pretension by the guiding carriage as well as forces pushing down, pulling up and pushing from the side.

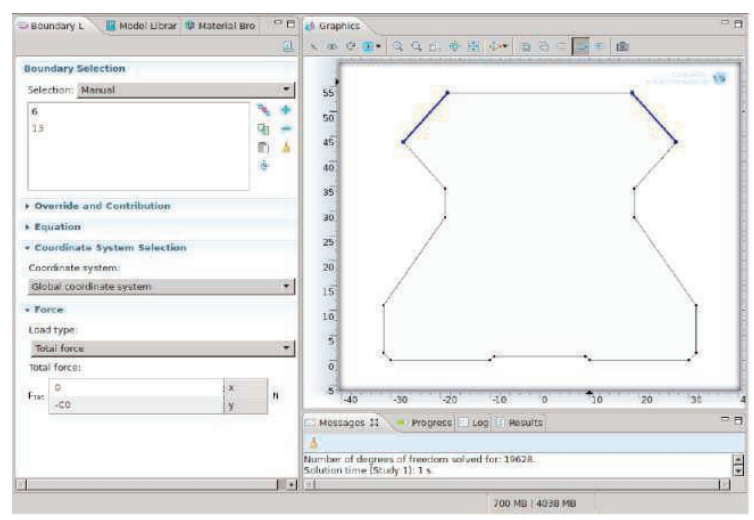

Fig. 9. Definition of a force pushing down on the rail. 


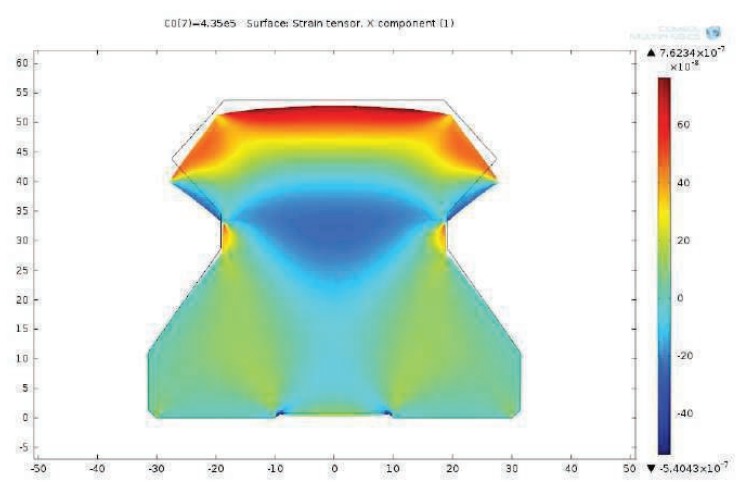

Fig. 10. Strain and material deformation during a load of $\mathrm{C}_{0}$.

Subsequently, the strain of the material was analyzed. The following figures show the results for the largest rail, size 65 . Figure 10 shows a typical result which corresponds to the definition of a force as depicted in figure 9. The static load rating $\mathrm{C}_{0}$ is the highest force that may act on the system without leaving plastic deformation of the rail or the rolling elements.

Figure 10 shows that the highest positive deformation is on the top surface of the rail. This edge will be subject of the following examinations. The next three figures show the strain tensor along this edge. In figure 11, the force is pushing down, in figure 12 it is pulling up and in figure 13 it pushes from the left to the right. The different lines represent a load of $\mathrm{C}_{0}$ (black) and fractions $(1 / 2,1 / 5,1 / 10,1 / 20,1 / 50$ and $1 / 100)$ of $\mathrm{C}_{0}$.

It can be seen, that not only the magnitude of the force, but also the direction can be detected by measuring the strain on the top surface of the rail. For forces pushing down, the strain is positive with two local maxima between $5 \mathrm{~mm}$ and $10 \mathrm{~mm}$ from the edge. For forces pulling up, the strain becomes negative with a maximum plateau in the middle of the rail. Finally, a force pushing from the side results in an asymmetrical strain distribution along the top surface of the rail. Similar simulations were carried out for Bosch Rexroth and THK rails and show a similar behavior.

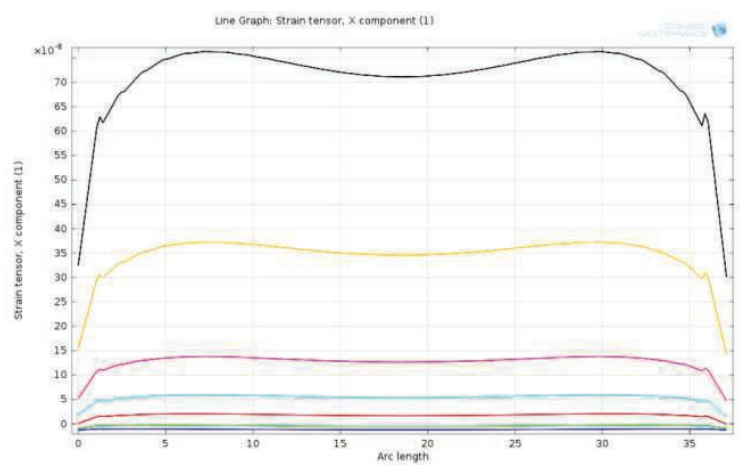

Fig. 11. Strain on the top edge of the rail during a load of $C_{0}$ pushing down on the rail.

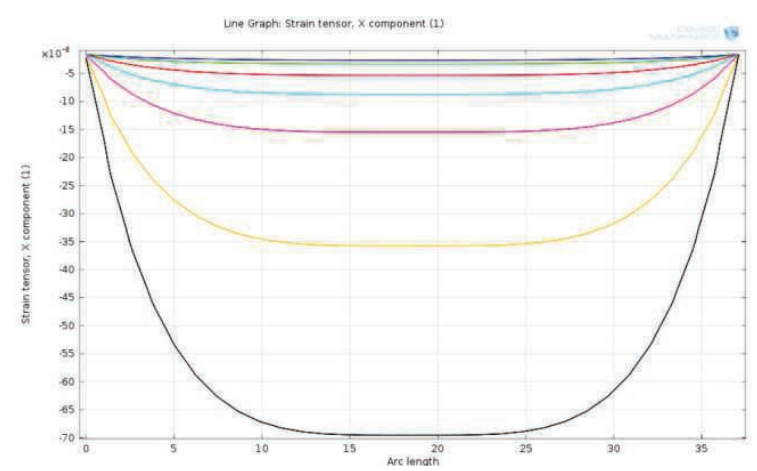

Fig. 12. Strain on the top edge of the rail during a load of $C_{0}$ pulling up the rail.

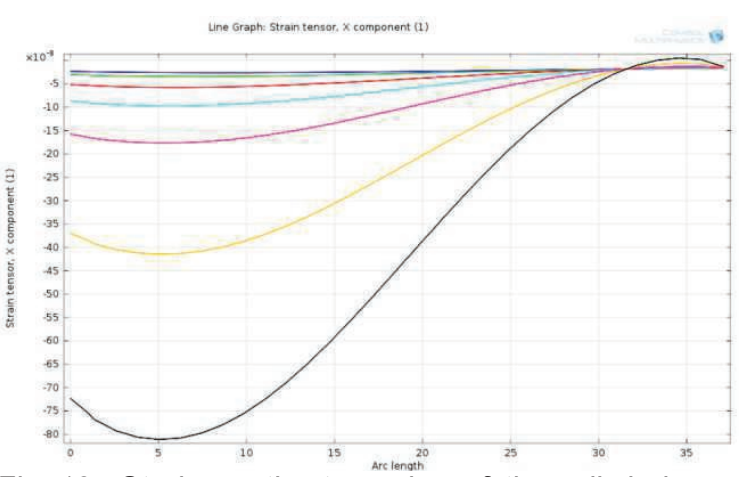

Fig. 13. Strain on the top edge of the rail during a load of $\mathrm{C}_{0}$ pushing from the left to the right.

To verify these theoretical results practically, three strain gauges were attached to the surface of a size 25 ball guide by Bosch Rexroth (figure 14). The guiding carriage was then slid on top of the sensor elements and a force was applied by a hydraulic cylinder. The force was measured by a load cell and the strain gauges were connected to a Wheatstone bridge. The supply voltage for the bridge was $5 \mathrm{~V}$ DC. A Keithley multimeter was used to measure the unamplified bridge voltage. Both values, force and bridge voltage, were recorded in a custom Labview program.

Figure 15 shows the bridge voltage during a load rising from $0 \mathrm{kN}$ to $5 \mathrm{kN}$. The sensitivity is approx. $5 \mu \mathrm{V} / \mathrm{kN}$.

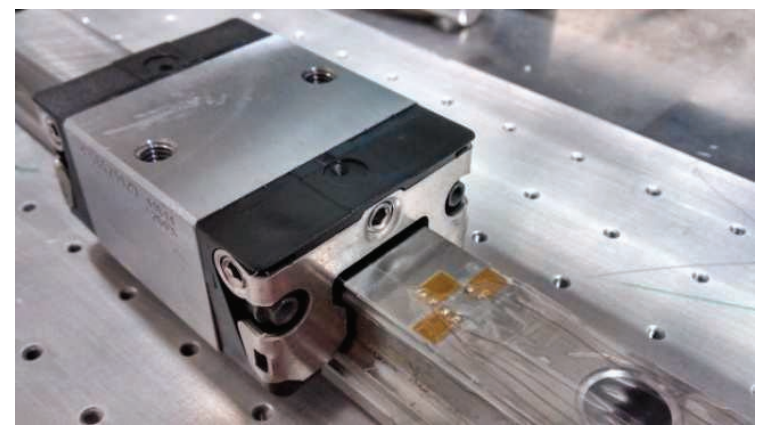

Fig. 14. Three strain gauges on the rail of a linear guide next to guiding carriage. 


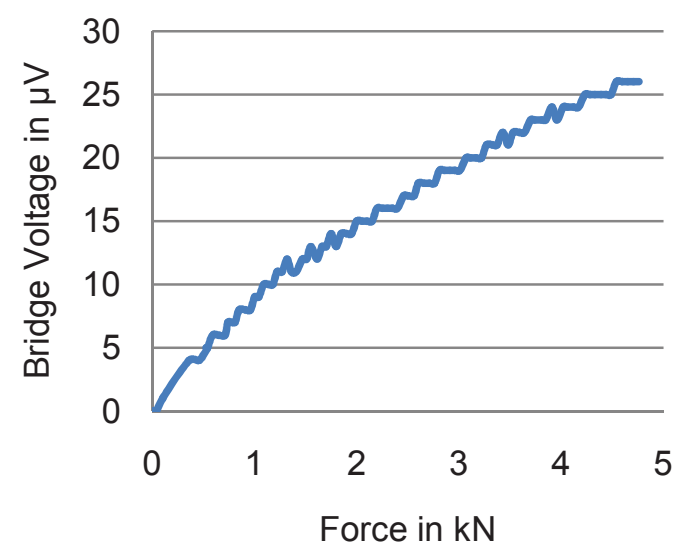

Fig. 15. Bridge voltage of the middle strain gauge during a force pressing down on the guide carriage.

The same measurement was conducted again, but this time a metal block on a wedge was placed on the guiding carriage. This results in different magnitudes for the signal of the left and right strain gauges on the rail. While both signals rise from $0 \mathrm{kN}$ to $1 \mathrm{kN}$, the signal of the left strain gauge decreases while the signal of the right strain gauge continues to rise. This shows that a moment around the long axis of the rail can be measured.

Further work will need to go into the application of sensorial surfaces instead of glued-on strain gauges to improve the durability of the sensor elements. Additionally, a compact read-out and interpretation unit will be developed.

\section{Conclusions}

Cyber-physical systems and integrated sensors will change automation and condition monitoring systems significantly in the future. Five different use cases were presented for different scenarios of component-based monitoring. Furthermore, first results for a monitoring system for linear guides were presented and show promising results for further developments.

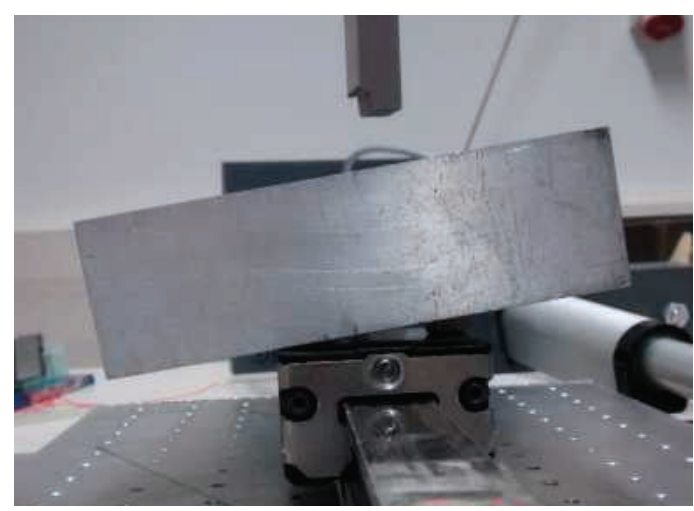

Fig. 16. Metal block on a wedge.

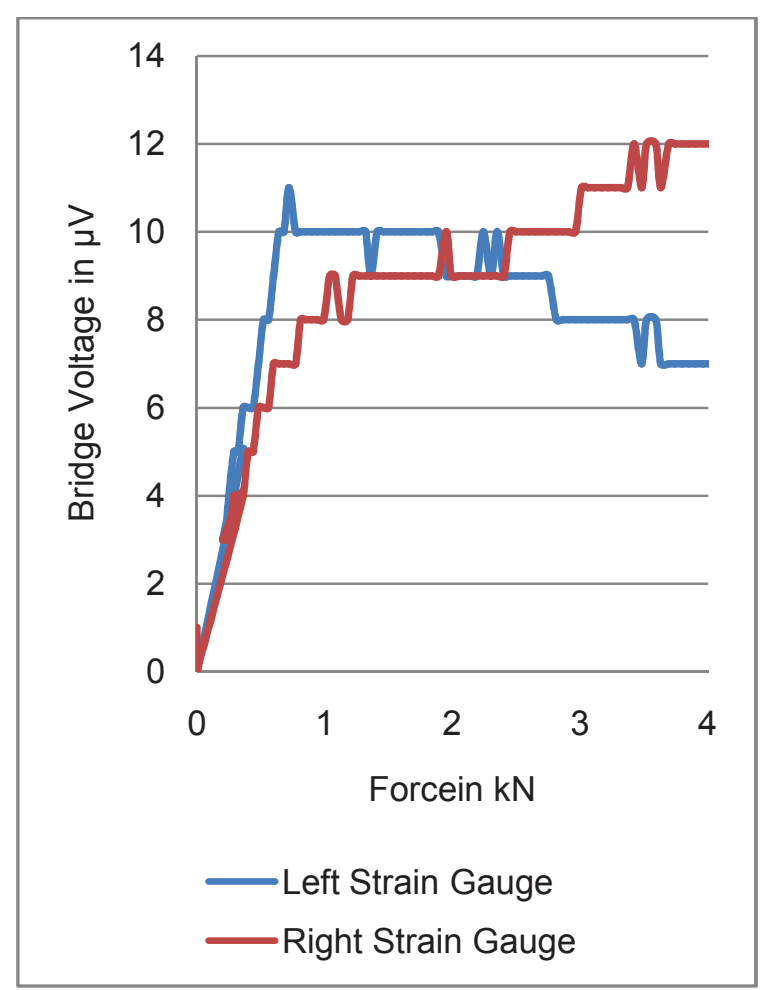

Fig. 17. Bridge voltage of the left and right strain gauges during a force pressing down on a wedged metal block on the guiding carriage.

\section{References}

[1] Herman, Pentek, Otto, Design Principles for Industrie 4.0 Scenarios, Working Paper No. 01/2015, Technische Universität Dortmund, (2015); http://www.snom.mb.tudortmund.de/cms/de/forschung/Arbeitsberichte/D esign-Principles-for-Industrie-4_0-Scenarios.pdf

[2] Kagermann, Wahlster, Helbig, Umsetzungsempfehlungen für das

Zukuntsprojekt Industrie 4.0, (2013); www.bmbf.de/pubRD/Umsetzungsempfehlungen _Industrie4_0.pdf

[3] Davis et al., Smart manufacturing, manufacturing intelligence and demand-dynamic performance, Computers \& Chemical Engineering 47, 145-156 (2012); doi:10.1016/j.compchemeng.2012.06.037

[4] Dumstorff, Lang, Integration without disruption: The basic challenge of sensor integration, IEEE Sensors Journal 14, 2102-2111 (2014); doi: 10.1109/JSEN.2013.2294626

[5] Winkelmann et al., Development of a strain sensor for rolling contact loads, Proceedings of Transducers 2011, Beijing, China, IEEE, 2011

[6] Winkelmann, Lang, Sensorial surfaces Embedding sensor structures into the surface of materials, IEEE Sensors Journal, 2078-2083 (2013); doi: doi: 10.1109/JSEN.2013.2290134 